\title{
FATORES DE RISCO DA SEPSE EM PACIENTES QUEIMADOS
}

\author{
RISK FACTORS OF SEPSIS IN BURNED PATIENTS
}

\author{
Jefferson Lessa S. de Macedo, TCBC-DF ${ }^{1}$; Simone Corrêa Rosa²; \\ Kátia Cilene Soares de Macedo ${ }^{3}$; Cleudson Castro ${ }^{4}$
}

\begin{abstract}
RESUMO: Objetivo: Os avanços no tratamento de queimados têm reduzido as taxas de mortalidade e melhorado a qualidade de vida das vítimas de queimaduras. Entretanto, a sepse continua sendo um desafio e umas das principais causas de óbito no queimado. O objetivo deste trabalho é investigar, através de um estudo caso-controle, os fatores de risco da sepse em pacientes queimados. Método: $\mathrm{O}$ estudo caso-controle foi conduzido durante 12 meses, compreendendo os pacientes que foram tratados em regime de internação hospitalar na Unidade de Queimados do Hospital Regional da Asa Norte (HRAN), Brasília-DF. Resultados: Quarenta e nove $(19,4 \%)$ pacientes tiveram sepse, de um total de 252 queimados internados na Unidade de Queimados durante o período do estudo. Eles tiveram um ou no máximo três episódios de sepse durante a internação, totalizando 62 episódios.Vinte e seis (53,1\%) eram homens e a média de idade foi de 21,9 $\pm 18,9$ anos (variação de um a 89 anos). A superfície corporal queimada dos pacientes que tiveram sepse variou de sete a $84 \%$, com uma média de $37,7 \pm 18,4 \%$, sendo significativamente superior aos controles. As principais bactérias causadoras de sepse foram Staphylococcus aureus (46,5\%), Staphylococcus coagulase negativo (20,7\%), Acinetobacter baumannii $(12,1 \%)$ e Enterobacter cloacae $(12,1 \%)$. Trinta $(61,2 \%)$ pacientes tiveram seu primeiro episódio de sepse na primeira semana de internação. Quanto aos fatores de risco para a ocorrência de sepse, destacam-se os seguintes, conforme seu poder de associação "odds ratio": o uso de três ou mais cateteres, a presença de duas ou mais complicações, a superfície corporal queimada $\geq 30 \%$, o agente chama aberta e o sexo feminino. No geral, a taxa de letalidade por sepse foi de $24,5 \%$. Conclusão: Um melhor conhecimento dos fatores de risco da sepse no paciente queimado permite o tratamento precoce dessa complicação, com antibioticoterapia sistêmica adequada, contribuindo para reduzir a morbidade e a mortalidade desses pacientes (Rev. Col. Bras. Cir. 2005; 32(4): 173-177).
\end{abstract}

Descritores: Queimaduras; Sepse; Fatores de risco; Infecção hospitalar

\section{INTRODUÇÃO}

Não existem estudos nacionais que apontem o número real de vítimas de queimaduras, mas, cerca de um milhão de reais por mês é o valor médio gasto pelo Ministério da Saúde com a internação destes pacientes ${ }^{1}$. Nos Estados Unidos, ocorrem dois milhões de queimaduras por ano e, embora a maioria dessas queimaduras sejam pequenas, 100.000 pacientes necessitam internação em centros de tratamento de queimados, e 5.000 desses pacientes morrem ${ }^{2,3}$.

Apesar do desenvolvimento de agentes antimicrobianos tópicos e sistêmicos, os avanços no suporte nutricional e o uso de técnicas cirúrgicas de excisão de tecidos desvitalizados e enxertia precoce da área queimada, a sepse continua representando um grande desafio e umas das principais causas de óbito no queimado ${ }^{3-5}$. A sepse ocorre quando o equilíbrio entre o hospedeiro e os microrganismos é rompido. Um dos principais fatores que alteram a defesa do hospedeiro, expondo-o aos patógenos, é a destruição da barreira mecânica da pele, favorecendo a invasão de germes por via linfática ou sangüínea.
Nas queimaduras, além da destruição da barreira epitelial, a presença de proteínas degradadas e tecidos desvitalizados proporcionam um excelente meio para o desenvolvimento e proliferação de microrganismos. Além disso, a obstrução vascular por lesão térmica dos vasos dificulta a chegada de antibióticos e de componentes celulares do sistema imune na área queimada ${ }^{6}$.

Outros fatores também favorecem a sepse no queimado, como a imunossupressão decorrente da lesão térmica, a possibilidade de translocação bacteriana gastrointestinal, a internação prolongada e o uso inadequado dos antibióticos, levando ao surgimento de bactérias com multiresistência antimicrobiana. $\mathrm{O}$ uso de cateteres, sondas e tubos, ou seja, procedimentos invasivos diagnósticos e terapêuticos que acabam alterando as defesas naturais do hospedeiro contra a infecção ${ }^{7}$, também contribuem para o desenvolvimento da sepse no paciente queimado.

O objetivo deste trabalho é investigar através de um estudo caso-controle os fatores de risco para o desenvolvimento de sepse no paciente queimado.

1. Mestre em Medicina pela Universidade de Brasília (UnB); Membro Titular da Sociedade Brasileira de Cirurgia Plástica (SBCP); Cirurgião Plástico do Hospital Regional da Asa Norte, Secretaria de Saúde do Distrito Federal, Brasília, DF.

2. Mestre em Medicina pela UnB; Membro Titular da SBCP; Cirurgiã Plástica do Hospital Regional da Asa Norte, Secretaria de Saúde do Distrito Federal, Brasília, DF.

3. Acadêmica de Medicina da Universidade Católica de Brasília.

4. Professor Adjunto de Doenças Infecto-Parasitárias da UnB.

Recebido em 20/12/2004

Aceito para publicação em 08/04/2005

Conflito de interesse: nenhum

Fonte de financiamento: nenhuma

Trabalho realizado na Unidade de Queimados do Hospital Regional da Asa Norte (HRAN) Brasília - DF. 


\section{MÉTODO}

O estudo foi conduzido durante doze meses compreendendo os pacientes que foram tratados em regime de internação hospitalar na Unidade de Queimados do Hospital Regional da Asa Norte (HRAN), Brasília-DF, no período de $1^{\circ}$ de junho de 2001 a 31 de maio de 2002. Não foram incluídos os pacientes atendidos no serviço de emergência do hospital que foram tratados a nível ambulatorial.

$\mathrm{O}$ atendimento inicial e os critérios de internação adotados na Unidade de Queimados do Hospital Regional da Asa Norte de Brasília já foram descritos anteriormente ${ }^{8}$.

Os pacientes considerados casos preencheram os seguintes critérios: quadro clínico compatível com sepse e hemocultura positiva. As manifestações clínicas da sepse incluíam dois ou mais sinais da síndrome da resposta inflamatória sistêmica (hipertemia $>38^{\circ} \mathrm{C}$ ou hipotermia $<36^{\circ} \mathrm{C}$; leucocitose $>12.000$ células $/ \mathrm{mm}^{3}$ ou leucopenia $<4.000$ células $/ \mathrm{mm}^{3}$ ou $>10 \%$ de bastonetes; taquicardia; taquipnéia) associada ou não a disfunções orgânicas, manifestando-se através de acidose lática, hipotensão grave, oligúria, alteração cardíaca não justificada (por exemplo: aparecimento de terceira bulha), invasão bacteriana de tecidos não queimados, trombocitopenia, azotemia não explicada ou deterioração do estado mental ${ }^{9}$.

Os controles foram escolhidos entre os pacientes queimados internados no HRAN no mesmo dia que um caso de sepse era detectado. Os controles foram pacientes internados na mesma enfermaria do caso ou em enfermarias vizinhas.

A proporção foi de dois controles para um caso. Não houve qualquer tipo de pareamento, pois o sexo e a idade, por exemplo, foram avaliados como fatores de risco para o desenvolvimento da sepse no queimado. Foram excluídos os controles que viraram casos durante a internação.

As hemoculturas foram colhidas por punção venosa, com técnica asséptica, utilizando material estéril e meios de cultura específicos padronizados para leitura em um sistema automatizado disponível no Hospital Regional da Asa Norte. Não foram colhidas hemoculturas através de cateteres e a limpeza da pele foi feita através de composto iodado. Foram colhidas duas amostras de locais diferentes, evitando áreas queimadas. Procurou-se colher as hemoculturas no início do pico febril ou durante a febre.

O sangue foi colhido de forma a respeitar a proporção de um mililitro de sangue para cada cinco a dez mililitros de meio de cultura (1:5 a 1:10). As hemoculturas foram processadas no Bact Alert 120 â e no Microsacan Walkway 96 â, aparelho que é conhecido pela rápida detecção do crescimento bacteriano levando a um diagnóstico precoce.

As informações sobre os fatores de risco e outras variáveis descritivas foram obtidas pelo pesquisador com a vítima ou com o responsável usando questionário e exame físico do paciente. Os fatores de risco estudados incluiram: a idade, o sexo, a superfície corporal queimada, o agente causador da queimadura (líquido quente, chama aberta, eletricidade, agente químico), a tentativa de auto-extermínio, o uso de três ou mais cateteres ou sondas (tubo endotraqueal, cateter venoso profundo, sonda vesical, sonda nasoenteral, sonda nasogástrica, cateter para oxigenioterapia), a presença de duas ou mais complicações (hematológica, renal, pulmonar, cardíaca, digestiva, hidroeletrolítica, neurológica ou amputações) e a procedência

Esse trabalho foi realizado sob aprovação do Comitê de Ética em Pesquisa da Secretaria de Saúde do Distrito Federal tendo como base a Resolução 196/96 CNS/MS (Processo número 003/2002).

A associação entre os possíveis fatores de risco e a ocorrência de sepse foram expressas em "odds ratio" (OR), que é considerado um estimador do risco relativo. Os intervalos de confiança de $95 \%$ para o OR foram reportados. Quando necessário, as proporções foram comparadas através do teste do qui-quadrado ou teste exato de Fisher. As diferenças foram consideradas significativas quando o valor do "p" associado ao teste do qui-quadrado era $<0,05$. Para esses cálculos estatísticos foi usando o pacote do "SPSS 10.0 for Windows".

\section{RESULTADOS}

Quarenta e nove $(19,4 \%)$ pacientes tiveram sepse de um total de 252 internados na Unidade de Queimados durante o período do estudo. Eles apresentaram um ou no máximo três episódios de sepse durante a internação, totalizando 62 episódios. Dos 49 pacientes com sepse, $26(53,1 \%)$ eram homens e a média de idade foi $21,9 \pm 18,9$ anos (variação de um a 89 anos). A chama aberta foi a causa predominante das queimaduras, sendo o agente responsável pela queimadura em 43 $(87,8 \%)$ dos casos de sepse, seguidos por escaldamento em cinco pacientes $(10,2 \%)$ e lesão elétrica em um paciente (Tabela 1).

Quarenta (81,6\%) pacientes tiveram um único episódio de sepse, e nove $(18,4 \%)$ tiveram até três. Dos 62 episódios de sepse, $58(93,5 \%)$ foram bacterianos e quatro $(6,5 \%)$ foram por Candida sp. Dos 58 episódios de sepse bacteriana, $39(67,2 \%)$ foram devidos a Gram- positivos e $19(32,8 \%)$ a Gram-negativos. Os sítios de origem dos 62 episódios de sepse foram: a ferida queimada em 15 (24,2\%) episódios, o pulmão em quatro $(6,1 \%)$, o cateter vascular em três $(4,8 \%)$ e a origem desconhecida ou não determinada em 40 (64,5\%) episódios. Trinta $(61,2 \%)$ pacientes tiveram seu primeiro episódio de sepse na primeira semana de internação, $14(28,6 \%)$ na segunda e cinco $(10,2 \%)$ na terceira semana ou mais de internação.

As principais bactérias causadoras de sepse foram Staphylococcus aureus (46,5\%), Staphylococcus coagulase negativo (20,7\%), Acinetobacter baumannii (12,1\%), Enterobacter cloacae (12,1\%), Klebsiella pneumoniae (8,6\%), e Pseudomonas aeruginosa (6,9\%).

A superfície corporal queimada dos pacientes que tiveram sepse variou de sete a $84 \%$ com uma média de $37,7 \pm$ $18,4 \%$, sendo significativamente superior aos controles (Tabela 1).

A idade, a procedência (Distrito Federal ou outro Estado), a tentativa de auto-extermínio $(14,3 \%$ x 9,8\%, $\mathrm{p}=0,42)$ e a ocorrência de acidente doméstico $(59,2 \%$ x 52,9\%, $\mathrm{p}=0,49)$ como causa da queimadura não mostraram diferenças significativas entre os grupos (casos $\mathrm{x}$ controles). 
Tabela 1 - Fatores de risco para sepse em pacientes queimados internados no Hospital Regional da Asa Norte, Brasília, DF.

\begin{tabular}{|c|c|c|c|c|c|c|c|}
\hline FATORES & Casc & $n=49$ & Contr & es $n=102$ & $\mathbf{p}$ & OR & IC \\
\hline & $\mathbf{n}$ & $(\%)$ & $\mathbf{n}$ & $(\%)$ & & & \\
\hline Sexo & & & & & & & \\
\hline Masculino & 26 & $(53,1)$ & 73 & $(71,6)$ & - & & $-\quad-$ \\
\hline Feminino & 23 & $(46,9)$ & 29 & $(28,4)$ & 0,016 & 2,46 & $1,21-5,01$ \\
\hline SCQ* média $(\%)$ & & $=18,4$ & & $=14,3$ & $<0,0001$ & - & - \\
\hline $\mathrm{SCQ} * \geq 30 \%$ & & & & & & & \\
\hline Sim & 30 & $(61,2)$ & 20 & $(19,6)$ & $<0,0001$ & 6,47 & $3,04-13,77$ \\
\hline Não & 19 & $(38,8)$ & 82 & $(80,4)$ & - & - & - \\
\hline Causa & & & & & & & \\
\hline Chama aberta & 43 & $(87,8)$ & 65 & $(63,7)$ & 0,002 & 4,08 & $1,59-10,49$ \\
\hline Escaldamento & 5 & $(10,2)$ & 30 & $(29,4)$ & - & - & - \\
\hline Elétrica & 1 & $(2,0)$ & 6 & $(5,9)$ & - & - & - \\
\hline Química & 0 & & 1 & $(0,9)$ & - & - & - \\
\hline Uso de três ou ma & & & & & & & \\
\hline Sim & 25 & $(51,0)$ & 8 & $(7,8)$ & $<0,0001$ & 12,24 & $4,91-30,52$ \\
\hline Não & 24 & $(49,0)$ & 94 & $(92,2)$ & - & - & - \\
\hline Duas ou mais com & & & & & & & \\
\hline Sim & 30 & $(61,2)$ & 16 & $(15,7)$ & $<0,0001$ & 8,49 & $3,87-18,59$ \\
\hline Não & 19 & $(38,8)$ & 86 & $(84,3)$ & - & - & - \\
\hline
\end{tabular}

* $S C Q=$ Superfície corporal queimada.

Dentre os fatores de risco para a ocorrência de sepse, destacam-se os seguintes, conforme seu poder de associação (OR): o uso de três ou mais cateteres, a presença de duas ou mais complicações, a superfície corporal queimada $\geq 30 \%$, o agente chama aberta e o sexo feminino (Tabela 1).

Doze $(24,5 \%)$ pacientes que apresentaram sepse morreram durante o estudo, com uma média de idade de 33 anos (um a 89 anos). A superfície corporal queimada desses pacientes variou de 17 a $84 \%$ (média $50 \%$ ) e sete $(58,3 \%$ ) desses pacientes tiveram mais de $50 \%$ da superfície corporal queimada. Houve 17 episódios de sepse nesses 12 pacientes, da internação no hospital até a morte, sendo isolado Gram-negativos em oito $(47,1 \%)$ episódios, Gram-positivos em sete $(41,2 \%)$ e fungos em dois $(11,8 \%)$ episódios.

A taxa de letalidade dos pacientes com sepse foi de $24,5 \%$ sendo significativamente superior aos $2,9 \%$ do grupo controle ( $<<0,0001$ ), com um "odds ratio" de 10,7.

\section{DISCUSSÃO}

Atualmente, a sobrevida dos pacientes portadores de queimaduras constitui um fato muito mais comum que em décadas passadas. Após os avanços no tratamento e no controle da reposição volêmica, a infecção emergiu como o principal risco para o paciente queimado. As lesões produzidas pelas queimaduras representam um local susceptível à colonização de organismos endógenos e/ou exógenos. O problema é complicado ainda mais pela depressão imunológica do hospedeiro, que é proporcional à extensão da lesão. Na queimadura, a progressão de simples colonização para infecção invasiva e sepse é dependente de fatores relacionados ao paciente, ao microrganismo ou a fatores iatrogênicos ${ }^{10}$.
No nosso trabalho, $81,6 \%$ tiveram um único episódio de sepse, sendo que $89,8 \%$ tiveram o primeiro episódio dentro de duas semanas de internação. Esses dados são comparáveis aos encontrados por Bang et al ${ }^{5}$. A superfície corporal queimada dos casos foi significativamente superior aos controles, contribuindo para uma maior taxa de letalidade dos casos, pois segundo Wolf et al $^{11}$, a extensão da queimadura é um fator preditor de letalidade.

O sítio da queimadura foi a porta de entrada mais provável para sepse, confirmado por outros estudos ${ }^{5,12-14}$, mas, em uma grande percentagem dos casos de sepse, o sítio de origem da sepse não foi detectado, conforme já reportado anteriormente ${ }^{13}$. Isso se deve provavelmente à dificuldade de se determinar constantemente a flora predominante na pele, na árvore traqueobrônquica, nos cateteres vasculares ou nas sondas.

Dos 58 episódios de sepse bacteriana, $67,2 \%$ foram devidos a Gram-positivos e 32,8\% devidos a Gram-negativos. A freqüência maior de sepse por gram-positivos já foi documentada por outros autores, destacando-se $S$. aureus. O número maior de episódios de sepse por $S$. aureus pode ser devido ao fato que as Unidades de Queimados representam a principal fonte dessa bactéria. Dezoito por cento das infecções que ocorrem no paciente queimado são atribuídas a essa bactéria. Normalmente, os fatores predisponentes são a internação prolongada e o uso de antibióticos sistêmicos, e a transmissão é geralmente realizada de um paciente para outro através da colonização das mãos ou das narinas dos profissionais de saúde, o ambiente, as soluções de sulfadiazina de prata e os tanques de hidroterapia ${ }^{5,15-19}$.

Apesar de P. aeruginosa já ter sido considerada o principal agente etiológico de sepse em queimados ${ }^{4}$, observamos uma frequiência baixa dessa bactéria como causa de sepse $(6,9 \%)$ já confirmada por outros estudos ${ }^{5,13,17}$. Isso deve-se 
ao fato que $P$. aeruginosa está competindo na colonização das queimaduras com outras bactérias mais adaptadas ou fungos que requerem menos nutrientes para sobreviver como $A$. baumannii e Candida sp, e ao uso de terapia sistêmica efetiva anti-Pseudomonas ${ }^{6,20,21}$.

Acinetobacter baumannii foi a terceira bactéria mais isolada nos casos de sepse em queimados após $S$. aureus e Staphylococcus coagulase negativo. É uma das poucas bactérias Gram-negativas que normalmente podem ser isoladas na pele humana, na garganta ou nas secreções de pessoas sadias. Está progressivamente sendo responsabilizada por infecções hospitalares, necessita de poucos nutrientes e são altamente resistentes aos antimicrobianos. A transmissão ocorre através das mãos e roupas dos profissionais de saúde e do ambiente contaminado ${ }^{21}$. Dezoito por cento dos pacientes infectados ou colonizados por A. baumannii desenvolvem bacteremia ou sepse ${ }^{20}$.

A observação feita nesse estudo que $12,1 \%$ dos episódios de sepse foram devidos a Acinetobacter baumannii é importante, pois sua emergência pode estar ligada ao uso extensivo de antibióticos de largo espectro, resultando na seleção de patógenos hospitalares, ou seja, a pressão seletiva do uso de antibióticos ${ }^{20,22}$.

Os fatores de risco para sepse em pacientes queimados, em ordem decrescente de poder de associação (OR), foram: o uso de três ou mais cateteres, a presença de duas ou mais complicações, a superfície corporal queimada $\geq 30 \%$, o agente chama aberta e o sexo feminino (Tabela 1). O uso de três ou mais cateteres ou sondas como importante fator de risco para sepse se deve ao rompimento das barreiras de proteção do indivíduo, favorecendo a colonização e infecção. Porém, muitas vezes os pacientes não podem ficar sem esses cateteres ou sondas, pois é através deles que recebem sua medicação ou nutrição, tão importantes para a recuperação. Entretanto, o uso desses cateteres ou sondas não deve ser prolongado, devendo ser retirados logo que possível, devido ao risco de sepse.

A presença de duas ou mais complicações também se mostrou um fator de risco importante e que caracteriza a gravidade do paciente. Portanto, por exemplo, um paciente que apresente uma complicação hematológica (anemia ou plaquetopenia) associada a uma complicação hidroeletrolítica (hiponatremia ou hipoglicemia) apresenta um risco oito vezes maior de apresentar sepse que um paciente em que não se observe duas ou mais complicações. Ocorrendo sepse, esse paciente apresenta um risco 11 vezes maior de morrer (Tabela 1). Esses dados são importantes e muito úteis na prática clínica, respondendo a questões nunca antes aventadas na literatura médica desse assunto.

A superfície corporal queimada igual ou maior de $30 \%$ também é um fator de risco para sepse. Os pacientes com áreas extensas de queimadura são graves e de difícil manejo clínico e cirúrgico, e apresentam maior letalidade ${ }^{8,11}$.

A chama aberta foi a principal causa das queimaduras tanto no grupo da sepse quanto nos controles. Além disso, o paciente cuja causa da queimadura foi a chama aberta apresenta um risco quatro vezes maior de desenvolver sepse, devido ao fato dessas queimaduras serem mais profundas e extensas que aquelas provocadas por escaldamento, predispondo a uma colonização maior da ferida e aumentando a possibilidade de sepse.

O sexo feminino como fator de risco para sepse é controverso, pois o sexo não é preditor de mortalidade em queimados ${ }^{11}$. Porém, no Brasil, devido à facilidade de acesso ao álcool líquido, não observada em outros países, é muito freqüente que as tentativas de auto-extermínio sejam realizadas por mulheres com o uso de álcool, apresentando uma maior taxa de letalidade, com significância estatística ${ }^{8}$. Nas residências brasileiras é muito comum encontrar um frasco de álcool líquido usado para limpeza doméstica, o que facilita as tentativas de auto-extermínio com o uso deste tipo de inflamável.

A taxa de letalidade por sepse verificada nesse estudo de $24,5 \%$ é comparável aos $29 \%$ observado por Bang et al..$^{5}$, e pode ser menor, desde que sejam observados alguns fatores, tais como a avaliação clínica e microbiológica contínuas, apropriada antibioticoterapia, cuidados de nutrição, cobertura precoce da ferida e diminuição do tempo de internação ${ }^{7,23}$

Portanto, para o controle efetivo da sepse em Unidades de Queimados é de suma importância o conhecimento da etiologia e dos fatores de risco. Além disso, deve-se ressaltar que futuros estudos abordando a resistência bacteriana e medidas auxiliares de controle de infecção cruzada serão fundamentais para prevenção, controle e tratamento dos casos de sepse em pacientes queimados.

\section{ABSTRACT}

Background: The advances of the treatment of burns have decreased the mortality rate and improved the quality of life of burned patients. Our objective is to analyse, through a case-control study, the risk factors of sepsis in burned patients. Methods: This casecontrol study included cases which were treated as in-patients at the Burn Unit of Hospital Regional da Asa Norte, Brasilia, Brazil, during an one year period. Results: Forty-nine (19.4\%) patients had sepsis amongst 252 cases admitted to the Burn Unit during the period of study. They had one to three septic episodes amounting to a total of 62 . Twenty-six (53.1\%) were males and the mean age was 22 years (range one year old to 89 years old). The total body surface area burned from the cases of sepsis varied from seven to $84 \%$ with a mean of $37.7 \pm 18.4 \%$, was significantly higher than controls. The most common bacteria isolated from the blood culture of the cases of sepsis were Staphylococcus aureus (46.5\%), Staphylococcus coagulase negative (20.7\%), Acinetobacter baumannii (12.1\%) and Enterobacter cloacae (12.1\%). Thirty (61.2\%) patients had their first septic episode either earlier or by one week postburn. The risk factors for sepsis were the use of three or more catheters, the presence of two or more complications, total body surface area burned $30 \%$, flame as cause of the burn and female sex. In general, the letality rate of sepsis was $24.5 \%$. Conclusion: The appropriate knowledge of risk factors of sepsis in burned patients permit early treatment of this complication, with an adequate systemic antibiotics, thus contributing to decrease the morbidity and letality of these patients.

Key words: Burns; Sepsis; Risk factors; Cross infection. 


\section{REFERÊNCIAS}

1. Ministério da Saúde do Brasil. Informe saúde. Ano VI, nº 152; 2002.

2. Brigham PA, McLoughlin E. Burn incidence and medical care used in the United States: estimate, trends, and data sources. J Burn Care Rehabil. 1996;17(2):95-107.

3. Nguyen TT, Gilpin DA, Meyer NA, et al. Current treatment of severely burned patients. Ann Surg. 1996;223(1):14-25.

4. Iribarren $\mathrm{OB}$, Perez JA, Valencia VC, et al. Infección en quemaduras: evaluación de antimicrobianos, escarectomía y balneoterapia. Rev Chil Cir. 1990;42(4):329-32.

5. Bang RL, Gang RK, Sanyal SC, et al. Burn septicaemia: an analysis of 79 patients. Burns. 1998;24(4):354-61.

6. Pruitt BA, McManus AT. The changing epidemiology of infection in burn patients. World J Surg. 1992;16(1):57-67.

7. Wurtz R, Karajovic M, Dacumos E, et al. Nosocomial infections in a burn intensive care unit. Burns.1995;21(3):181-4.

8. Macedo JL, Rosa SC. Estudo epidemiológico dos pacientes internados na Unidade de Queimados: Hospital Regional da Asa Norte, Brasília, 1992-1997. Brasília Méd. 2000;37(3/4):8792.

9. Bone RC, Balk RA, Cerra FB, et al. Definition for sepsis and organ failure and guidelines for the use of innovative therapies in sepsis. The ACCP/SCCM Consensus Conference Committee. American College of Chest Physicians/Society of Critical Care Medicine. Chest. 1992;101(6):1644-55.

10. Barreto MX, Leonardi DF, Silva MA. Infecção em queimaduras: estudo da flora predominante na UTI-Queimados do Hospital de Pronto-Socorro de Porto Alegre. Rev Bras Terap Intensiva. 1998;10(4):177-80.

11. Wolf SE, Rose JK, Desai MH, et al. Mortality determinants in massive pediatric burns. An analysis of 103 children with $>$ or $=80 \%$ TBSA burns ( $>$ or $=70 \%$ full-thickness $)$. Ann Surg. 1997;225(5):554-69.

12. Muller MJ, Herndon DN. The challenge of burns. Lancet. 1994; 343(8891):216-20.
13. Lesseva M. Central venous catheter-related bacteremia in burn patients. Scand J Infect Dis. 1998;30(6):585-9.

14. Pruitt BA, McManus AT, Kim SH, et al. Burn wound infections: current status. World J Surg. 1998;22(2):135-45.

15. Blot SI, Hoste EA, Vandewoude K, et al. Staphylococcal septicaemia in burns. Burns. 2001;27(2):203.

16. Cook N. Methicillin-resistant Staphylococcus aureus versus the burn patient. Burns. 1998;24(2):91-8.

17. Song W, Lee KM, Kang HJ, et al. Microbiologic aspects of predominant bacteria isolated from the burn patients in Korea. Burns. 2001;27(2):136-9.

18. Embil JM, McLeod JA, Al-Barrak AM, et al. An outbreak of methicillin resistant Staphylococcus aureus on a burn unit: potencial role of contaminated hydrotherapy equipment. Burns. 2001;27(7):681-8.

19. Holder IA, Neely AN. Fear of MRSA-potential for future disaster. Burns. 1998;24(2):99-103.

20. Cisneros JM, Reyes MJ, Pachon J, et al. Bacteremia due to Acinetobacter baumannii: epidemiology, clinical findings, and prognostic features. Clin Infect Dis.1996;22(6):1026-32.

21. Sengupta S, Kumar P, Ciraj AM, et al. Acinetobacter baumannii - an emerging nosocomial pathogen in burns unit Manipal, India. Burns. 2001; 27(2):140-4.

22. Wisplinghoff H, Perbix W, Seifert H. Risk factors for nosocomial bloodstream infections due to Acinetobacter baumannii: a casecontrol study of adult burn patients. Clin Infect Dis.1999;28(1):59-66.

23. Macedo JL, Rosa SC, Castro C. Sepsis in burned patients. Rev Soc Bras Med Trop. 2003;36(6):647-52

Endereço para correspondência:

Jefferson Lessa soares de Macedo

SQS 213 Bloco "H" Apto 104. Asa Sul.

70 292-080- Brasília- DF

Telefax: (61) 3278415

Email: jlsmacedo@yahoo.com.br 